\title{
Towards a Theory of Graphic Medicine
}

\author{
Sathyaraj Venkatesan \& Anu Mary Peter \\ ${ }^{1}$ Associate Professor, Department of Humanities and Social Sciences, National Institute of \\ Technology (NIT), Tiruchirappalli, Tamil Nadu, India-620015, orcid.org/oooo-0oo3-2138- \\ 1263. sathya@nitt.edu, \\ ${ }^{2}$ Research Scholar, Department of Humanities and Social Sciences, National Institute of \\ Technology, Tiruchirappalli, Tamil Nadu, India-620015, anupeterthekk@gmail.com, \\ orcid.org/oooo-0001-6740-8252
}

First published July 20, 2019

\begin{abstract}
As a constructive derivative of several altruistic movements, such as narrative medicine, medical humanities and health humanities, graphic medicine is a nonconformist ideological inverse to the absolutism of medical knowledge. Exposing the limitations of biomedicine, which prioritises the voice of the physician, graphic medicine provides an inclusive approach towards medical conditions through its perspectival richness and experiential realities. Formulated as a means to express the voice of the marginalised community of sufferers, graphic medicine expedites creative and forceful articulation of the narratives of patients and caregivers, which were hitherto discarded as inauthentic by the existing medical system. As a wholesome methodology that equally values the subjective experiences of patients, physicians and caregivers, graphic medicine provides numerous ways of representing affective truths about illness conditions. Thus, by creating a mutually beneficial field of study for both biomedicine and humanities by yoking together medicine and comics, graphic medicine fosters an empathetic attitude towards human conditions. The present chapter traces the evolution of graphic medicine from the historical nexus of comics and medicine, through narrative medicine, to health humanities, and introduces graphic medicine as a cultural alternative to the existing paradigms of medical knowledge. Additionally, this chapter also aims to rationalise the unique aspects and cultural roles of graphic medicine-pedagogical, therapeutic, and community formational.
\end{abstract}

Keywords: graphic medicine, Comics, illness, Biomedicine.

\section{Narrative Medicine: Understanding the Ordeals of Illness}

Owing to the supremacy of the empiricist approach in medical narratives, the ideological rift between specialist medical knowledge and the unvoiced illness repertoires of individuals widened. Discerned as the outcome of various attempts during the 1970s to formulate "a hermeneutic position" on the patient (Hyden 51), pioneered by Rita Charon, narrative medicine emerged as a radical approach towards the expression of illness experience and as a reaction against the oppressive authoritarianism of medical knowledge. Referring to its significance, Charon (1265) contends: "I first used the phrase 'narrative medicine' in 2000 to refer to clinical practice fortified by narrative competence-the capacity to recognize, absorb, metabolize, interpret, and be moved by stories of illness." Fervidly criticizing biomedicine for its lack of appreciation for non-medical narratives or personal accounts of illness experiences, narrative

(c) AesthetixMS 2019. This Open Access article is published under a Creative Commons Attribution Non-Commercial 4.0 International License (http://creativecommons.org/licenses/by-nc/4.o/), which permits non-commercial re-use, distribution, and reproduction in any medium, provided the original work is properly cited. For citation use the DOI. For commercial re-use, please contact editor@rupkatha.com. 
medicine successfully shattered the illusion of medical science's primacy. Thus, offering "healthcare professionals with practical wisdom in comprehending what patients endure in illness and what they themselves undergo in the care of the sick" (Charon vii), narrative medicine foregrounded for the first time that without a practice of listening to the sufferer's subjective experiences medical science is just insufficient in facilitating a holistic experience of recovery to patients.

Narrative medicine prioritized patients' expression of illness experience in their own words as opposed to the fact-focused empirical autocracy of medical science. Charon's approach taught health care professionals to listen "expertly and attentively to extraordinarily complicated narratives" of patients or caregivers, expressed through "words, gestures, silences, tracings, images, laboratory test results, and changes in the body" (4). Thus, urging to foster a culture of listening, narrative medicine goaded the medical community "to understand as best they can the ordeals of illness, to honor the meanings of their patients' narratives of illness, and to be moved by what they behold so that they can act on their patients' behalf" (Charon 3). While bringing patients' experiences to the vanguard of medical science, narrative medicine also offered myriad ways of understanding illness as a disruptive experience rather than as a clinical entity, thereby successfully tethering the ideologically differing poles of experience and empiricism. In other words, emphasizing the need to assimilate tacit knowledge into medical diagnosis and treatment, narrative medicine effectually underscored the significance of acknowledging the voice of the sufferer.

\section{Health Humanities: Reconfiguring Biomedicine through Arts}

Anchored on the philosophical construct that "it is through arts and humanities that we can fully grasp the meaning of events and experiences in healthcare", health humanities provide a collective understanding of illness experiences (Crawford 8). Thus enabling sufferers and caregivers to express their experiences through various artistic means, health humanities enhance critical reflection and facilitate the health care profession to progress beyond the restraining framework of factual analysis and empirical evidence. In essence, by providing insights into the complexities of human experiences through visual and verbal modes of expression, health humanities blur the boundaries between arts and biomedicine by yoking them together. Interestingly, health humanities is also crafted as a singularly potent approach to illuminate the experiences of those who are largely left out by narrative medical movements, for instance, informal caregivers, healers, motivators, unpaid caretakers and other paraprofessionals. As Crawford et al. observe: "there are areas where there is yet relatively little scholarship but where the arts and humanities are poised to make a contribution, such as exploring the experiences of carers, reconnoitring the work of clients and practitioners in roles beyond the relatively narrow set which have so far been explored" (Health Humanities 12).

As a wholesome approach that values the experiences of patients, physicians, and caregivers, health humanities delineate illness experiences through arts and various genres of verbal or visual expression. Some of the popular representations of illnesses and pain across various artistic media are Frida Kahlo's painting The Wounded Deer, which is about her harrowing medical experiences. Similarly, Michelangelo's La Pieta is a nonpareil illustration of ineffable trauma sculpted in marble. Likewise, John Keats's poems that are soused in melancholic lure are achingly beautiful expressions of his TB experience. In music, Demi Lovato's songs share similar 
thematic moorings of illness. Marvelyn Brown's HIV experience chronicled as The Naked Truth (2008) reinforces the theme of illness representation in textual narratives. Considering movies as yet another medium of powerful expression of illness experience, Ron Howard's A Beautiful Mind (2001) is an intense depiction of schizophrenia. Interestingly, Bill T. John's dance performances choreographed with illness themes are scintillating instances of illness evinced through performative art forms. In photography, NGOs such as Keith Berr's Flashes of Hope, which utilises creative photography for sharing cancer experiences, attempt to alter the way children with terminal illnesses see themselves. While the artworks above are examples of the representation of illness and pain across verbal or visual media, there are comics on illness experiences, which bring together both verbal and visual media. When various other media provide limited portrayals of subjective human experiences, comics' engagement with illness is idiosyncratic and extra forceful as it creatively deploys the medium-specific strengths of both visual and verbal media of expression.

\section{Graphic Medicine: Definition and Scope}

Situated well within the praxis of health humanities, graphic medicine efficaciously delineates haptic and tacit dimensions of human experience. As the authors confirm in the Graphic Medicine Manifesto, graphic medicine is a unique means of "interrogating the representation of physical and emotional signs and symptoms within the medium" (Czerwiec 1). With the commencement of a website - graphicmedicine.org - in 2007 , graphic medicine as an academic discourse was established by Ian Williams, an English physician. The graphic medicine community comprises other notable academicians and health-care practitioners, namely M. K. Czerwiec, Michael J. Green, Kimberly R. Myers, Susan M. Squier, Scott T. Smith, among others. After gaining ideological and pragmatic fruition through substantial academic interventions, graphic medicine began to be celebrated as the iconoclastic nexus of comics and medicine. In the seminal indenture Graphic Medicine Manifesto, Williams observes the correlation of graphic medicine with the aims of narrative medicine thus: "graphic medicine combines the principles of narrative medicine with an exploration of the visual systems of comic art, interrogating the representation of physical and emotional signs and symptoms within the medium" (1). As Squier contends "while narrative medicine focuses on the textual and verbal, graphic medicine can access those aspects of illness and medicine that we experience visually and spatially, as enduring, if intractable, aspects of the patient's experience" (Czerwiec 46).

Put differently, exploiting the medium-specific uniqueness of comics such as panels, gutters, iconic images, speech and thought balloons, graphic medicine narrates as well as visibilises the experiential realities of illness conditions. The hybrid vigour of comics facilitates graphic medicine to be the ideal approach to make appalling and near-death experiences of illness visceral and immediate. With the synergetic interflow of both verbal and visual components in comics, graphic medicine creatively embraces the lived experiences of sufferers and idiosyncratically manifests their subjective experiences. Thus, perspectival richness and the potential to spatiotemporally map the tacit experiences formulate graphic medicine as one of the finest genres for achieving therapeutic, community-building and pedagogical goals of autobiographical narratives. 
With the primary objective of sharing and legitimizing affective truths constituted in illness and suffering, graphic medicine emphasizes the phenomenological and intimate aspects of such experiences and emotions. Exposing the limitations of extant ideological systems through creative deconstruction, graphic medicine challenges the attitude of submissiveness that is cultivated towards biomedicine. Occasionally, by providing a holistic understanding of medical regimes and practices, graphic medicine also complements medical knowledge. As Williams contends, "authors of graphic memoirs of illness, by depicting their conditions and experiences, create valuable new knowledge" (Czerwiec 133). Thus, by offering numerous explications of biomedical approaches as well as its lack of consociation with the experience of the sufferer, graphic medicine validates itself as an alternate source of knowledge.

\section{Graphic Medicine as a Disruptive Movement}

Exposing medical knowledge as limited, graphic medicine positions itself as an alternative to medical knowledge. As Squier contends, while foregrounding "the disruptive urgency of graphic memoirs", graphic medicine also interrogates the "epistemological authority of the medical profession" (Configurations 150). By prioritising the experiential truths of sufferers and caregivers, graphic medicine expropriates the monopoly of medical knowledge and thereby acts as a redefined discourse on medical experiences. Through the conscious integration of medical knowledge with "the continuities and discontinuities in attitudes and beliefs both within and beyond the health sciences" (Gilman 299), graphic medicine emerges as an exemplary paradigm of knowledge to reappraise the ascendancy of medical knowledge in the twenty-first century. Graphic medicine, as Squier observes, "is a movement for change that challenges the dominant methods of scholarship in healthcare, offering a more inclusive perspective of medicine, illness, disability, caregiving and being cared for" (Czerwiec 2). By exposing the narrative limitations of expert medical knowledge, graphic medicine has an overhauling effect on the socio-cultural understanding of medical experience. Thus, by expanding a range of subject positions, graphic medicine also introduces analytic practices that are hitherto unacknowledged by the medical system. Emphatically questioning empirical knowledge with subjective truths, graphic medicine facilitates a necessitous revamping of the defeatist biomedical and cultural perceptions of illness conditions. Thus, yoking together "the personal with pedagogical, the subjective with the objective-the image with the text", graphic medicine productively brings together a plethora of dissenting voices, concepts and practices (Czerwiec 3). Dominant graphic medical narratives such as Dana Walrath's Aliceheimer's: Alzheimer's Through the Looking Glass (2013), David Wojnarowicz's 7 Miles a Second (1996), Peter Dunlap-Shohl's My Degeneration, A Journey Through Parkinson's (2015), show how graphic medicine functions as a cultural and ethical alternative to the extant medical knowledge by disrupting the existing notions of illness realities through experiential accounts.

Published in the year 2013, Dana Walrath's Aliceheimer's: Alzheimer's Through the Looking Glass (2013) (hereafter Aliceheimer's) is a phenomenal example of how graphic medicine functions as an ethical alternate fount of knowledge to the hegemonic and hardly subjective medical discourse. Critiquing and reconfiguring the ruthless and reductionist approach of biomedicine and socio-cultural systems towards degenerative memory diseases, Walrath takes a creative and innovative digression to foreground "dementia as a different way of being, as a window into another reality" (4). Rather than reiterating the biomedical understanding of degenerative cognitive impairments that are depicted insensitively through horror images, Walrath uses 
images of magic and mirth to present Alzheimer's as an occasion to disinter the previously unexplored realms of the self. Denuding medical science as "a collection of beliefs and practices", Walrath argues for the dire necessity to rescind the medical system by saying that "it is a cultural system and that, like any human-made system, it has its flaws" (4). Interestingly, tethering her mother's steady decline into oblivion with the adventurous descent of Alice into a surreal world in Lewis Carroll's Alice in Wonderland, Walrath not only dispels the stigma associated with AD but also creates an aura of innocuousness and invulnerability to it. In essence, Aliceheimer's creatively performs the stated objective of graphic medicine, which is to "challenge accepted conventions of scholarship" (Czerwiec 3), and triumphs as one of the major graphic memoirs to celebrate that which is condemned by the empirical notions of medical knowledge.

In a similar vein, originally published by Vertigo Verite in 1996 and republished later in 2012 by Fantagraphics, 7 Miles $A$ Second concerns the traumatic AIDS experiences of David Wojnarowicz against the backdrop of various social-political injustices that occurred during the AIDS crisis in the 1980s. Attempting to rescript the dominant socio-cultural, medical as well as religious attitudes towards AIDS-affected individuals, the graphic memoir is an assertive expression of the distinctive potentials of graphic medicine in "exploring taboo or forbidden areas of illness and healthcare" (Czerwiec 3) by offering "voice to those who are often not heard", as stated in the Graphic Medicine Manifesto (Squier and Czerwiec 2). Delineating the ignominious societal perspectives regarding AIDS proliferated through popular media, Wojnarowicz's experiences unravel the resultant stigmatising and subsequent victimisation of AIDS patients as anti-social and as human jeopardies to be excluded from mainstream society. Contrasting the available discourse on AIDS with the heretofore redacted knowledge about the patient's lived experience of the illness, 7 Miles A Second exposes certain dual standards prevalent in medical and socio-religious institutions. Exploiting the rhetorical features of the stream of consciousness technique and the structural affordances of the medium of comics, the graphic narrative is an idiosyncratic venting of the repressed experiential truth of an AIDS patient. Critiquing the visual representations of AIDS-affected individuals as "bed ridden and alone" (n.p.), Wojnarowicz condemns their compassionless cultural denigration of AIDS sufferers as "outsider", "carrier" and "infected" (n.p.) in accordance with the conventional standards of social acceptance. Conclusively, the memoir strategically deploys AIDS as "a weapon to enforce the conservative agenda" of dominant institutional powers (n.p.).

\section{Graphic Medicine as an Educational Tool}

Although it divulges medical information regarding illness conditions, graphic medicine is beyond the measured precincts of pedagogical comics. While the objectives of educational comics are restricted to the dissemination of information or creating awareness among the masses, graphic medicine has multifarious aims, such as sharing subjective experiences, attaining therapeutic or cathartic objectives, altruism and community formation, among others. Misapprehended often as educational comics, graphic medical narratives are marked by their thematic diversity, narrative intricacy, and stylistic ingenuity. Anchoring on a single theme, educational comics are mostly correlated to the clinical specificities of a particular illness, whereas graphic pathographies negotiate multiple themes that are not only related to medical aspects but also the subjective socio-cultural and psychological experience of the illness. Antithetical to the lucid and linear narrative arc of educational comics, graphic medical narratives are exceedingly subjective recollections of disruptive and traumatic illness experiences that are 
often narrated non-linearly. Narrated from the third person in instructional and uncomplicated clinical language, educational comics seldom deploy decorative linguistic and semantic features, unlike graphic pathographies, which are usually narrated from the first, second and third person. Stylistically, educational comics are illustrative, simplistic and therefore unchallenging, whereas graphic pathographies are a mostly complex manifestation of highly creative experimentation with the art form. Further, educational comics have a specific target audience, whereas graphic pathographies attract wider attention from not only patients, but also doctors, other health-care providers, caregivers, and even general readers. Having said that the principal objective of graphic medicine is not to educate the reader, it is used as a means to achieve clinical and non-clinical objectives in medical schools as part of the professional pollarding of future doctors.

Owing to the tremendous capacity of the genre to communicate traumatic experiences, graphic medicine is utilised as an academic tool to enhance "doctoring skills" (Czerwiec 73) in American medical schools. Introduced by Michael Green (Professor in the Departments of Humanities and Internal Medicine) in the Penn State College of Medicine in 2009, the "Graphic Storytelling and Medical Narrative" course for the fourth-year medical students is a manifest of the cultural impact of graphic medicine. Comics help in enhancing their core cognitive skills "empathy, communication, clinical reasoning, writing, attention to nonverbal cues, and awareness of physician bias" (Green 774). While reading comics enriches medical students' communicative skills, deductive reasoning, behavioural skills and diagnostic capabilities, then making comics enables them to "think creatively without inhibitions" (Czerwiec 72). Facilitating a vivid documenting of their harrowing experiences, comics creation helps medical trainees to divulge their seemingly unacknowledged experiences of victimisation by seniors and patients during medical education.

An analysis of the comic rendition of fourth-year medical students' experiences reveals that students often portray "workplace [as] dank dungeons; patients as ghosts that haunted physicians who had treated them impersonally; supervising physicians as fiendish, foul-mouthed monsters; and themselves as sleep-deprived zombies walking through barren apocalyptic landscapes" (George and Green 2345). Graphic narratives like Vita Perseverat by Ashley L. Pistorio, Not My Forever by Stephanie Bennett (2016), Lou DiSalvo's Delirium (2016), Whatever Floats Your Boat by Shane Lavin (2014) created by fourth-year medical students evince how graphic medicine, when used as an academic contrivance, creates effective prospects for students to resolve and represent their determinative experiences. Put together, graphic medicine when integrated into the medical school curriculum encourages future health-care professionals not only to comprehend and perceive patients' experience but also to pronounce and portray their own trauma as well. Accordingly, in spite of the ideological difficulty in categorically defining graphic medicine under the rubrics of educational comics, graphic medicine is utilised as an academic tool, and graphic medical narratives occasionally communicate clinical and other essential information as well.

Graphic medical narratives such as Peter Dunlap-Shohl's My Degeneration: A Journey Through Parkinson's and Monsters by Ken Dahl are unique examples of graphic pathographies that have educational qualities as well. My Degeneration: A Journey through Parkinson's (2015) by Peter Dunlap-Shohl, published by Pennsylvania State University Press, is an honest and humorous account of the author's journey through Parkinson's disease since 2002. Diagnosed with Parkinson's at the age of forty-three, Dunlap in his memoir offers a creative blend of 
information and introspection about the disease. As Dunlap observes, the memoir is a mélange of "personal incidents, medical facts, waiting-room lore, compelling analogies and a solid story arc" (Dunham Alaska Artist's Graphic Novel About Parkinson's Disease Part of Innovative Series of Medical Texts). Together with the portrayal of the transformations that he underwent when his life's natural cadence was unexpectedly disrupted by Parkinson's, Dunlap's memoir is also a fascinating account of creatively dealing with it. Dunlap's memoir imaginatively provides advantageous information about some positive adaptive techniques such as "walking backward", "throwing toes", "pigeon-toed walk", "the straddle step", "the flying leap", and "rag kicker's dream" to name a few. Thus, aiming to empower people with similar helplessness occasioned by Parkinson's, the graphic memoir alerts them to comprehend the disruption that the illness has caused and also to gain control over the altered life through self-reformulation, hope, and certain techniques. In a similar vein, as a semi-fictional graphic memoir about Ken Dahl's self-disgust on contracting herpes, Monsters published by Secret Acres in 2009, is "an exercise in the grotesque that manages to yield important clinical and emotional information" (Czerwiec 122). Commenting on the socio-cultural construction of illnesses, Monsters visualises the shame and social ostracising experienced by those who suffer from sexually transmitted diseases. Monsters as a graphic memoir with educational value, interfuses the creative complexities of graphic memoirs that are exemplified through intricate metaphors and imageries with beneficial information about herpes.

\section{Graphic Medicine as Therapy}

Having emerged out of the burgeoning demand for documenting disregarded subjective truths, graphic medicine provides a visual language of self-expression to translate the manifold experiences of suffering. In the conclusion to the Graphic Medicine Manifesto, Juliet McMullin states that "graphic medicine is an inspiration" in such a way that it reminds individuals about the necessity to narrate experiences, especially illness experiences, in order to suture their own lives and to help others also (Czerwiec 169). Effectuating an unprejudiced venting of emotions, which leads to meaning-making and reconciliation, the creation of comics enables the sufferer to logically assimilate the incoherent experiences into a narrative. Drawing impetus from the spatiotemporal attributes of the medium of comics, graphic medicine manifests the fragmentary rhythm of illness experience through panels and gutter spaces. As Williams contends, "comics demand reader participation-inviting readers to empathize with a subject by entering its world and seeing through its eyes-and enable the reader to gain insight from the vicarious experience" (127). Invited by the author into the intra-diegetic spaces of the narrative to perform the role of a listener, witness or spectator, the reader shares the author's fragmented vision and experiences the similar trail of thoughts and emotions that the comics pages relay. While narrating traumatic experiences, graphic medicine deftly articulates the innermost self through pictures rather than words. Deploying the technique of embodiment, the medium enables authors to represent themselves and their trauma with approximation. Exploring "embodiment as a medium of experience" (Couser 294) to visibilize the ineffable traumatic experiences, graphic medicine deploys the medium's ability to encapsulate private trauma into an expression of the incommunicable, thereby foregrounding its therapeutic potential.

Essentially, by valuing lived body experiences and their intense emotive dimensions, graphic medicine espouses the expression of the intimate and ineffable in graphic pathographies. Since most graphic pathographies relive traumatic episodes from the patient's/caregiver's life, 
such scripting and illustrating of painful episodes turn into latitudes, which facilitate them to reflect and comprehend their untranslatable pain. For instance, in Stan Mack's Janet $\mathcal{E}$ Me, published in 2004 by Simon and Schuster Paperbacks, Mack attains self-reconciliation and emotional release through the making of an illustrated memoir about the traumatic experience of witnessing his partner Janet's breast cancer and her subsequent death. Vindicating his choice to graphically illustrate the traumatic experiences of the duo, Mack attests that the process of the making of the memoir strengthened him to transmute the agony into a language that allowed him to "move beyond the experience" (Pennebaker 13). The cathartic purpose of graphic medicine is thus evident in Mack's remark in the "Author's Note" to Janet $\mathcal{E} M e$ : "[I]n the process, I discovered that sketching our neighbourhood and apartment, portraying Janet and myself together during the good times and bad, depicting us talking to each other, helped me put painful memories to rest" (ix). Put differently, Mack found the illustration and narration of his memories as the right inducement for purging his painful emotions. The graphic memoir thus becomes a repository of his traumatic past, which found expression and meaning by purging Mack of its incomprehensibility and psychic perturbation.

In the conclusion to the Graphic Medicine Manifesto, Sarah Leavitt testifies the therapeutic properties of graphic medicine by stating that "graphic medicine has comforted and sustained me in hard times: both reading others' work and creating my own" (Czerwiec 168). Published in 2012 by Skyhorse, Tangles, A Story about Alzheimer's, My Mother, and Me is an emotionally upheaving graphic memoir about Leavitt's experience of caring for her mother, Midge, who was suffering from Alzheimer's disease. Characterized by scant and crude drawings, Tangles is a graphic manifestation of attaining emotional stability followed by a tragic loss by visually scripting the traumatic experiences. Leavitt shares with the readers in the introductory note that the memoir is a "story ... pieced together from [] memories" (n.p.). Carefully created through four years of "writing and drawing" (n.p.), Leavitt acknowledges in the introduction to Tangles as well as in the conclusion to the Graphic Medicine Manifesto how the process of making the memoir proved to be a creative anodyne to her. Associating graphic medicine with metaphors of consolation and comfort such as "homemade soup" or "blanket", Tangles is a visual attestation of the therapeutic effect of graphic medicine.

\section{Graphic Medicine and Community Formation}

Graphic medical memoirs, as Williams observes, "stem precisely from the need to express oneself and, possibly, to challenge the 'medical' authority from which the author feels excluded" (Czerwiec 129). Interestingly, graphic pathographies facilitate the formation of a brotherhood of sufferers through the sharing of subjective experiences of illness and pain with visual immediacy. In the Graphic Medicine Manifesto, Squier states that "community building is a central strategy of graphic medicine" (53). Creatively utilising iconic images in their narratives, graphic pathographers "illuminate the universals of the human condition by revealing the particular" (Charon 9). In the conclusion of the Graphic Medicine Manifesto, comic artists such as Shelly Wall and John Swogger attest that graphic medicine provides "inspiration, community, and a voice" to sufferers who are often silenced by illness perceptions and also "a powerful way of talking about the human context of medical stories" (Czerwiec 172). Rivetingly, graphic medicine serves as a "growing community where many people [] [find] their voices" (Czerwiec 3) and also makes sufferers conscious that they are not alone. Thus, graphic medicine, which "represents multiple subjects with valid, and at times conflicting points of view and experience" (Czerwiec 2), also 
functions as an emotional community that appreciates and acknowledges myriad experiences and practices of healing and confronting illness. Some graphic memoirs such as Katie Green's Lighter Than My Shadow, and I Had a Black Dog by Matthew Johnstone reflect the community building objective of graphic medicine.

Critically acclaimed for the forceful visual narration of her struggles with anorexia and binge eating, Lighter Than My Shadow, published by Random House in 2013, is Green's first graphic narrative. As asserted in the blurb, the memoir is also "an exposure to those who are so weak they prey on the weak, and an inspiration to anybody who believes in the human power to endure towards happiness" (n.p). Bringing together the anorectic, bulimic and sexually abused, Green's memoir aspires to be inspirational by creatively manifesting multiple prospects of recovery for all. Portraying the progressive psychological path of her illness by negotiating her aversion to food and the consequent feeling of culpability, Green offers a factual and haptic narration of the cycles of recovery and relapse and survival from eating disorders. Delineating through haunting imageries the caustic aftermaths of sexual abuse and stigma associated with body image, Green alerts the reader to the psychological triggers of eating disorders. Reflecting on the intensity of her experiences, the graphic narrative deftly expresses Green's trauma through the visual iconography of a dark patch of scribble. Experimenting with multiple shades, panel borders and blank pages, Lighter is a memoir of recovery and restoration. In the conclusion to the Graphic Medicine Manifesto, Green attests the community formation capability of graphic medicine by stating that "I was met with only empathy and support. I met people from such a range of different backgrounds, many of whom became ... a community of support" (Czerwiec 167).

In similitude with Green's memoir, Johnstone's I Had a Black Dog is also a graphic memoir that effectively creates an emotional bond with those who are suffering from depression and thus an apt exemplar for graphic medical narratives' ability to form a community. Encapsulating with creativity and perfection one of the most common mental conditions, the memoir is a graphic formulation of the legendary black dog euphemism for depression coined by Winston Churchill. Metaphorically depicting his depression as a domineering dog with an intensely symbolic black colour, the author successfully portrays the experience of depression with accuracy, clarity, and authenticity. Through images, the author effectively communicates coping mechanisms and techniques to tame the beast and attain recovery. Underscoring the capability of graphic medicine to bring together those who are suffering, the author attests in his blog that publishing his experience in order to help others was one of the best things he has ever done.

\section{Conclusion}

Graphic medicine emerges at the crossroads of health humanities and narrative medicine, negotiates various illness experiences by transcending the boundaries of biomedicine and the cultural nuances on illness and health. Through its intrinsic yet authentic portrayal of subjective experiential truths from multiple perspectives, graphic medicine demonstrates how patients, caregivers and not just health-care professionals can contribute substantially to the holistic understanding of medical conditions. Utilising the structural nuances of the medium of comics, graphic medicine forges an intricate relationship between doctors, patients, and caregivers and thus creates an alternative knowledge system. Put differently, the partial representation of the experiences of the patient as a means of perpetuating and preserving the knowledge grids of 
medicine was destabilised with the advent of graphic medicine, which was receptive to all forms of experiential knowledge irrespective of source and power hierarchies. Cultivating a profound understanding of illness as a subjective experience, graphic medicine when used as a pedagogical tool enables effectual transmission of information and also enhances the clinical and communicative capabilities of future physicians when practised in medical school. In a similar vein, comics creation also aids medical students in expressing their medical school experience with creative scintilla. By creating safe narrative spaces to be self-reflective, graphic medicine enables authors to access their inner realms that are fragmented by traumatic experiences, thereby effectuating its therapeutic goals. Also, by allowing subjugated voices to be heard and suppressed perspectives to be shared, graphic medicine forms an emotional community by bringing together individuals of similar experiences, thereby extending empathy and support. While narrative medicine is limited in many ways to the verbal media, graphic medicine facilitates access to unutterable experiences of illnesses through verbal and visual methods. Further, comics is also "a powerful medium to bring biocultural analyses of medicine, as well as health humanities, to a wide audience" (Squier 2015, 46). In essence, considering its multi-layered strengths, graphic medicine is an intransigent aggregation of experiential as well as empirical truths.

\section{References}

Charon, R. (2006). Narrative medicine: Honoring the stories of illness. Oxford: Oxford University Press.

Couser, G. T. (1997). Recovering bodies: Illness, disability, and life writing. Madison: University of Wisconsin Press.

Crawford, P., Brown, B., Baker, C., Tischler, V., \& Abrams, B.(2015). Health humanities. London: Palgrave Macmillan.

Czerwiec, M.K., Williams, 1., Squier, S.M., Green, M.J., Myers, K.R., \& Smith, S.T. (2015). Graphic medicine manifesto. Pennsylvania: The Pennsylvania State University Press.

Dahl, K. (2009). Monsters. Jackson Heights, NY: Secret Acres.

Dunlap-Shohl, P.(2015). My degeneration: A journey through parkinson's. Pennsylvania: The Pennsylvania University Press.

George, D. R., \& Green, M. J. (2015). "Lessons learned from comics produced by medical students: Art of darkness." Journal of American Medical Association, 314(22), 2345-2346.

Gilman, S. (2011). Representing health and illness: Thoughts for the twenty-first century. Medical History. 55 (3): 295-300.

Green, K. (2013). Lighter than my shadow. London: Random House.

Hyden, L.C.(1997). "Illness and narrative." Sociology of Health E Illness. 19 (1): 48-69.

Johnstone, M. (2005). I had a black dog: His name was depression. Sydney: Pan.

Leavitt, S (2012). Tangles: A story about alzheimer's, my mother, and me. New York: Skyhorse.

Mack, S.(2004). Janet E me: An illustrated story of love and loss. New York: Simon \& Schuster.

Pennebaker, J.W.(2000). “Telling stories: The health benefits of narrative." Literature and Medicine. 19 (1): 318.

Refaie, E. E. (2012). Autobiographical comics: Life writing in pictures. Jackson: University Press of Mississippi.

Squier, S.M. (2008). "Literature and medicine, future tense: Making it graphic." Literature and Medicine. 27 (2): 124-152.

Walrath, D. (2013). Aliceheimer's: Alzheimer's through the looking glass. The Pennsylvania State University Press.

Wojnarowicz, D, James Romberger, Cook M. Van, and Eric Reynolds. (1996). 7 Miles a Second. Fantagraphics Books. 